\title{
THE
}

\section{Domination Game: A proof of the 3/5-Conjecture for Graphs with Minimum Degree at Least Two}

\author{
Michael A. Henning \\ William B. Kinnersley \\ University of Rhode Island, billk@uri.edu
}

Follow this and additional works at: https://digitalcommons.uri.edu/math_facpubs

\section{The University of Rhode Island Faculty have made this article openly available. \\ Please let us know how Open Access to this research benefits you.}

This is a pre-publication author manuscript of the final, published article.

Terms of Use

This article is made available under the terms and conditions applicable towards Open Access Policy Articles, as set forth in our Terms of Use.

\section{Citation/Publisher Attribution}

Henning, M. A. \& Kinnersley, W. B. (2016). Domination Game: A proof of the 3/5-Conjecture for Graphs with Minimum Degree at Least Two. SIAM J. Discrete Math., 30(1), 20-35. doi: 10.1137/140976935 Available at: https://doi.org/10.1137/140976935 


\title{
DOMINATION GAME: A PROOF OF THE 3/5-CONJECTURE FOR GRAPHS WITH MINIMUM DEGREE AT LEAST TWO
}

\author{
MICHAEL A. HENNING AND WILLIAM B. KINNERSLEY
}

\begin{abstract}
In the domination game on a graph $G$, the players Dominator and Staller alternately select vertices of $G$. Each vertex chosen must strictly increase the number of vertices dominated. This process eventually produces a dominating set of $G$; Dominator aims to minimize the size of this set, while Staller aims to maximize it. The size of the dominating set produced under optimal play is the game domination number of $G$, denoted by $\gamma_{g}(G)$.

In this paper, we prove that $\gamma_{g}(G) \leq 2 n / 3$ for every $n$-vertex isolate-free graph $G$. When $G$ has minimum degree at least 2 , we prove the stronger bound $\gamma_{g}(G) \leq 3 n / 5$; this resolves a special case of a conjecture due to Kinnersley, West, and Zamani [SIAM J. Discrete Math. 27 (2013), 2090-2107]. Finally, we prove that if $G$ is an $n$-vertex isolate-free graph with $\ell$ vertices of degree 1 , then $\gamma_{g}(G) \leq 3 n / 5+\lceil\ell / 2\rceil+1$; in the course of establishing this result, we answer a question of Brešar, Dorbec, Klavžar, and Košmrlj [Discrete Math. 330 (2014), $1-10$.$] .$
\end{abstract}

\section{INTRODUCTION}

Graph domination is an extensively studied area of graph theory on which numerous papers have been written (see [15] for an overview). We say that a vertex dominates itself and its neighbors; a dominating set in a graph $G$ is a set of vertices of $G$ that dominates all vertices in the graph. When studying domination on a graph $G$, one typically seeks the minimum size of a dominating set in $G$; this is known as the domination number of $G$, denoted $\gamma(G)$. In this paper, we study a game in which two agents collaboratively build a dominating set. In this game, one player aims to minimize the size of the dominating set produced, while the other aims to maximize it. This game, the domination game, was first introduced by Brešar, Klavžar, and Rall [5]. Several papers since have been written on the topic; see [2], [4], [6], [7], [17], and [20].

Formally, the domination game is a two-player game played on a host graph $G$. The players, Dominator and Staller, take turns adding vertices to a set $S$, which is initially empty. Every vertex added must strictly increase the number of vertices dominated by $S$. Eventually, $S$ becomes a dominating set of $G$. Dominator seeks to dominate the graph "efficiently"; as such, his goal is to minimize the size of the dominating set produced. Staller, on the other hand, aims to maximize the size of the set. When both players play optimally, the size of the resulting dominating set is the game domination number of $G$, denoted by $\gamma_{g}(G)$ (resp. $\gamma_{g}^{\prime}(G)$ ) when Dominator (resp. Staller) plays first.

The domination game belongs to the growing family of competitive optimization graph games. Broadly speaking, "competitive optimization" describes a process in which multiple agents with conflicting goals collaboratively produce some special structure in an underlying host graph. In the domination game, that structure is a dominating set, and the players' goals are completely antithetical: while Min wants to minimize the size of a dominating 
set, Max wants to maximize it. Thus, the domination game is a competitive optimization variant of the well-studied domination problem on graphs. One of the first and best-known competitive optimization parameters is the celebrated game chromatic number, in which the players collaboratively construct a proper coloring of the host graph. This parameter was first introduced by Gardner [12], and has seen extensive study [11, 16, 18, 22]). Recently, work has been done on competitive optimization variants of list-colouring [21, 23], matching [10], Ramsey theory [3, 13, 14], and more [1, 19].

Our focus in the present paper is a conjecture posted by Kinnersley, West, and Zamani in [20]. The 3/5-Conjecture states that if $G$ is an $n$-vertex isolate-free graph (that is, a graph having no vertices of degree 0 ), then $\gamma_{g}(G) \leq 3 n / 5$. (In fact, there are two "3/5Conjectures": one for general isolate-free graphs, and one for isolate-free forests. It is not known whether one implies the other.) In [20], the authors showed that the $3 / 5$-Conjecture holds when $G$ is an isolate-free forest of caterpillars. When $G$ is only required to be an $n$-vertex isolate-free forest, they showed that $\gamma_{g}(G) \leq 7 n / 11$; for general isolate-free graphs, they proved the weaker bound $\gamma_{g}(G) \leq\lceil 7 n / 10\rceil$. Recently, Brešar, Klavžar, Košmrlj, and Rall [4] verified the 3/5-Conjecture for all trees on at most 20 vertices, and listed those meeting the conjectured bound with equality (when $n=20$, there are only ten such trees). In addition, Bujtás [7] has proved the 3/5-Conjecture for isolate-free forests in which no two vertices lie at distance 4 , and has improved on the best known general upper bounds for isolate-free graphs and isolate-free forests [8].

In this paper, we make progress toward the $3 / 5$-Conjecture for general connected graphs. In Section 2, we first state an improved upper bound for $\gamma_{g}(G)$ : our Corollary 2.3 is that $\gamma_{g}(G) \leq 2 n / 3$ for every $n$-vertex isolate-free graph $G$. We next go on to establish the conjecture over the class of graphs with minimum degree at least 2. In Section 3, we show

that $\left|\gamma_{g}(G)-\gamma_{g}(H)\right| \leq|E(G) \triangle E(H)|+1$ whenever $G$ and $H$ are graphs on the same vertex set (where $\triangle$ denotes symmetric difference). This answers a question posed in [2]; moreover, we apply this result to give a partial answer to another question from [2]. Finally, we apply Theorem 3.4 to prove a bound (Corollary 3.5) that is, in some cases, stronger than Theorem 2.2.

\section{General Bounds}

We begin by proving that $\gamma_{g}(G) \leq 2 n / 3$ for every $n$-vertex connected graph $G$; while this does not establish the $3 / 5$-Conjecture, it does improve upon the bound $\gamma_{g}(G) \leq\lceil 7 n / 10\rceil$ established in [20]. Very recently, Bujtás [8] has claimed a proof of the stronger upper bound $\gamma_{g}(G) \leq 0.64 n$; we have opted to retain the proof of our weaker bound both for historical interest and to act as an introduction to some techniques used later in the section.

Before proceeding, we introduce some important concepts and notation. A partiallydominated graph is a graph in which we suppose that some vertices have already been dominated, and need not be dominated again to complete the game. For $G$ a graph and $S \subseteq V(G)$, we denote by $G \mid S$ the partially-dominated graph corresponding $G$ with the vertices in $S$ already dominated. We use $\gamma_{g}(G \mid S)$ (resp. $\gamma_{g}^{\prime}(G \mid S)$ ) to denote the number of turns remaining in the game on $G \mid S$ under optimal play when Dominator (resp. Staller) has the next turn. The following result from [20] often proves useful:

Theorem 2.1 ("Continuation Principle" - [20], Lemma 2.1). Let $G$ be a graph and let $A, B \subseteq V(G)$. If $B \subseteq A$, then $\gamma_{g}(G \mid A) \leq \gamma_{g}(G \mid B)$ and $\gamma_{g}^{\prime}(G \mid A) \leq \gamma_{g}^{\prime}(G \mid B)$. 
It sometimes happens that vertices and edges in a partially-dominated graph are irrelevant in that their presence or absence does not affect the game. We call a vertex $v$ saturated if $v$ and all of its neighbors have already been dominated. Given a partially-dominated graph $G$, the residual graph $G^{\prime}$ corresponding to $G$ is obtained from $G$ by deleting all saturated vertices and all edges joining dominated vertices; it is straightforward that $\gamma_{g}\left(G^{\prime}\right)=\gamma_{g}(G)$ and $\gamma_{g}^{\prime}\left(G^{\prime}\right)=\gamma_{g}^{\prime}(G)$. A reduced graph is one that is equal to its residual graph.

To simplify the arguments in this paper, we make a slight modification to the rules of the domination game: on his turns, we allow Dominator to play any vertex he wishes, regardless of whether or not he dominates any new vertices. (We even allow Dominator to play vertices that have already been played; technically, this means that we are now constructing a dominating multiset.) By the Continuation Principle, such moves are never in Dominator's best interests, so it does not hurt to allow this extra flexibility. Conversely, when we present a Dominator strategy, this rule change saves us the trouble of having to verify that all of Dominator's moves are "legal".

We are now ready to prove our first main result. This proof is relatively straightforward compared to those later in the paper, and serves both as a warm-up and a gentle introduction to the types of arguments we use later.

Theorem 2.2. Let $G$ be an isolate-free partially-dominated graph. If $G$ has $n$ vertices, of which $d$ are dominated and $s$ are saturated, then

$$
\gamma_{g}(G) \leq \frac{2 n-s-d}{3} \quad \text { and } \quad \gamma_{g}^{\prime}(G) \leq \frac{2 n-s-d+1}{3} .
$$

Proof. Without loss of generality, we may assume that $G$ is a reduced graph (since otherwise, we may operate instead on its residual graph). We use induction on $n-s$. For the base case, suppose that $G$ has $k$ components, each isomorphic to $K_{2}$. Then, $n-s=2 k$ and, since each component contains at least one undominated vertex, $d \leq k$. Moreover, in the remainder of the game, exactly one move gets made in each component. Thus $\gamma_{g}(G)=\gamma_{g}^{\prime}(G)=k \leq$ $(2 n-s-d) / 3$, so both bounds hold.

Assume now that $G$ has some component not isomorphic to $K_{2}$. Suppose Staller plays first. Staller's move must dominate at least one vertex (by the rules of the domination game) and saturate at least one (in particular, the vertex Staller played). Letting $G^{\prime}$ denote the resulting residual graph, we have

$$
\gamma_{g}^{\prime}(G)=1+\gamma_{g}\left(G^{\prime}\right) \leq 1+\frac{2 n-(s+1)-(d+1)}{3}=\frac{2 n-s-d+1}{3},
$$

where the first equality follows from optimality of Staller's move, and the subsequent inequality follows from the induction hypothesis applied to $G^{\prime}$. (Note that $G^{\prime}$ must be isolate-free, since every component of $G^{\prime}$ has at least one undominated vertex, and all undominated vertices in $G^{\prime}$ have the same degree as in $G$.)

Now suppose that Dominator plays first. Let $C$ be some component of $G$ not isomorphic to $K_{2}$. We have two cases to consider. In each case we specify Dominator's move, and we let $G^{\prime}$ denote the resulting residual graph.

Case 1: $C$ has a leaf. Let $v$ be any leaf of $C$ and let $u$ be its neighbor. If both $v$ and $u$ are undominated, then Dominator plays $u$; this dominates and saturates both $u$ and $v$. Now

$$
\gamma_{g}(G) \leq 1+\gamma_{g}^{\prime}\left(G^{\prime}\right) \leq 1+\frac{2 n-(s+2)-(d+2)+1}{3}=\frac{2 n-s-d}{3}
$$


where the first inequality holds because Dominator does at least as well playing optimally as he does by playing on $u$, and the second inequality follows from the induction hypothesis applied to $G^{\prime}$.

If instead $v$ is undominated but $u$ is dominated, then the choice of $C$ implies that $u$ has some undominated neighbor $t$. (Note that in a reduced graph, all neighbors of a dominated vertex must be undominated.) Dominator plays $u$, thereby dominating $t$ and $v$, and saturating $u$ and $v$. Thus

$$
\gamma_{g}(G) \leq 1+\gamma_{g}^{\prime}\left(G^{\prime}\right) \leq 1+\frac{2 n-(s+2)-(d+2)+1}{3}=\frac{2 n-s-d}{3} .
$$

The only remaining possibility is that $v$ is dominated but $u$ is not. If $u$ has an undominated neighbor $t$, then Dominator plays $t$, which dominates $t$ and $u$, and saturates $t$ and $v$. Once again, we have

$$
\gamma_{g}(G) \leq 1+\gamma_{g}^{\prime}\left(G^{\prime}\right) \leq 1+\frac{2 n-(s+2)-(d+2)+1}{3}=\frac{2 n-s-d}{3} .
$$

Finally, suppose that $u$ has no undominated neighbors. The choice of $C$ implies that $u$ has at least one neighbor other than $v$, say $t$. Dominator plays $t$, which dominates $u$ and saturates $t$ and $v$. Since $u$ had no undominated neighbors, this move also saturates $u$. Now

$$
\gamma_{g}(G) \leq 1+\gamma_{g}^{\prime}\left(G^{\prime}\right) \leq 1+\frac{2 n-(s+3)-(d+1)+1}{3}=\frac{2 n-s-d}{3} .
$$

Case 2: $C$ has no leaves. We consider three subcases.

Case 2a: some undominated vertex $v$ in $C$ has at least two undominated neighbors. Dominator plays $v$, which dominates at least three vertices ( $v$ and its undominated neighbors) and saturates $v$. Now

$$
\gamma_{g}(G) \leq 1+\gamma_{g}^{\prime}\left(G^{\prime}\right) \leq 1+\frac{2 n-(s+1)-(d+3)+1}{3}=\frac{2 n-s-d}{3} .
$$

We may thus assume that no undominated vertex in $C$ has two undominated neighbors.

Case 2b: some undominated vertex $v$ in $C$ has one undominated neighbor. Let $u$ be the undominated neighbor of $v$. By assumption, $u$ has no other undominated neighbors. Dominator plays $u$; this dominates and saturates both $u$ and $v$. Now

$$
\gamma_{g}(G) \leq 1+\gamma_{g}^{\prime}\left(G^{\prime}\right) \leq 1+\frac{2 n-(s+2)-(d+2)+1}{3}=\frac{2 n-s-d}{3} .
$$

Case 2c: $C$ contains no adjacent undominated vertices. Since $G$ is isolate-free, $C$ contains at least one pair of adjacent vertices, at least one of which must be dominated; let $v$ be a dominated vertex in $C$. Since $C$ has no leaves, $v$ must have at least two neighbors, both undominated. Let $u$ and $w$ be two undominated neighbors of $v$; by assumption, neither $u$ nor $w$ has any undominated neighbors. Dominator plays $v$, which dominates $u$ and $w$, and saturates $u, v$, and $w$. Now

$$
\gamma_{g}(G) \leq 1+\gamma_{g}^{\prime}\left(G^{\prime}\right) \leq 1+\frac{2 n-(s+3)-(d+2)+1}{3}=\frac{2 n-s-d-1}{3}<\frac{2 n-s-d}{3} .
$$

Theorem 2.2 yields the following result as a special case.

Corollary 2.3. If $G$ is an $n$-vertex isolate-free graph, then $\gamma_{g}(G) \leq \frac{2 n}{3}$ and $\gamma_{g}^{\prime}(G) \leq \frac{2 n+1}{3}$. 
We next verify the 3/5-Conjecture for graphs with minimum degree at least 2 . To do this, we use techniques similar to those used in proving Theorem 2.2. As before, we consider the more general family of partially-dominated graphs. We define a linking vertex to be a dominated vertex with at least two undominated vertices. The heart of our proof is the following lemma:

Theorem 2.4. Let $G$ be a reduced graph in which all undominated vertices have degree at least 2. If $G$ has $n$ vertices, of which $d$ are dominated, $s$ are saturated, and $\ell$ are linking vertices, then

$$
\gamma_{g}(G) \leq \frac{6 n-4 d-2 s+\ell}{10} \quad \text { and } \quad \gamma_{g}^{\prime}(G) \leq \frac{6 n-4 d-2 s+\ell+5}{10} .
$$

Before launching into the proof of Theorem 2.4, we develop the underlying intuition. Define the value of a move in the domination game to be $4 d^{\prime}+2 s^{\prime}-\ell^{\prime}$, where $d^{\prime}$ and $s^{\prime}$ denote the number of additional vertices dominated and saturated, respectively, by the move, and $\ell^{\prime}$ denotes the change in the number of linking vertices. For example, a move that dominates two vertices, saturates one, and creates one linking vertex would have value $4 \cdot 2+2 \cdot 1-1$; a move that dominates one vertex, saturates three, and reduces the number of linking vertices by two would have value $4 \cdot 1+2 \cdot 3+2$. The significance of the value will be formalized later, but essentially it provides a measure of "how good" the move is for Dominator. (We remark that linking vertices turn out to be problematic for Dominator, hence the negative weight on $\ell^{\prime}$ : we "penalize" Dominator for creating them, and "reward" him for eliminating them.)

In proving Theorem 2.4, when some player makes a move on $G$, we would like to inductively apply the claimed bounds to the resulting residual graph; we can then use the induction hypothesis to bound the length of the game on $G$. The value of a move represents the corresponding decrease in the numerators of the bounds claimed in Theorem 2.4 or, in other words, how much "stronger" the inductive bounds are than the original claimed bounds. Dominator aims to ensure that throughout the duration of the game, the average value of each move is at least 10. As it will turn out, Dominator is content to always play moves with value 15 or higher since, as the next lemma shows, Staller's ensuing move must have value at least 5 .

We next state two auxiliary lemmas that will aid in proving Theorem 2.4.

Lemma 2.5. If $G$ is a reduced graph without isolated vertices, then every move in the domination game on $G$ has value at least 5; moreover, every move with value 5 consists of playing at a dominated leaf whose undominated neighbor itself has at least two other undominated neighbors.

Proof. Let $G$ be a reduced graph without isolated vertices, and suppose a move is made at vertex $v$. Let $d^{\prime}$ and $s^{\prime}$ denote the number of vertices dominated and saturated, respectively, by this move, and let $\ell^{\prime}$ denote the change in the number of linking vertices. By the rules of the game, the move must dominate at least one vertex, so $d^{\prime} \geq 1$. Moreover, it must saturate at least one vertex - namely $v$ itself - and so, $s^{\prime} \geq 1$. Finally, the only vertices that could possibly become linking vertices are those dominated by the move, so $\ell^{\prime} \leq d^{\prime}$. Bounding the value of the move, we see that $4 d^{\prime}+2 s^{\prime}-\ell^{\prime} \geq 3 d^{\prime}+2 s^{\prime} \geq 5$, from which the first claim follows. 
In order for the move to have value 5 , we must have $d^{\prime}=1$ and $s^{\prime}=1$ and, moreover, the single vertex dominated by the move must become a linking vertex. In particular, the vertex dominated and the vertex saturated must be distinct. Hence $v$ must have already been dominated, and must have had only one undominated neighbor; that is, $v$ was a dominated leaf. In order for the neighbor to become a linking vertex, it must have had at least two other undominated neighbors. This establishes the second claim.

Before proceeding to the proof of the main result, we present another simple but useful lemma.

Lemma 2.6. Let $G$ be a reduced graph, and let $v$ be a dominated leaf in $G$ with undominated neighbor $w$. If all neighbors of $w$ are dominated, then

$$
\gamma_{g}(G-v)=\gamma_{g}(G) \quad \text { and } \quad \gamma_{g}^{\prime}(G-v)=\gamma_{g}^{\prime}(G)
$$

Proof. In the game on $G$, neither player need ever play $v$, since there is no difference between playing $v$ and playing $w$.

Lemma 2.6 permits a special sort of chicanery. Suppose some move in the domination game yields a dominated leaf $v$ with undominated neighbor $w$, all neighbors of which are dominated. By Lemma 2.6, deleting $v$ cannot affect the remaining length of the game. In such situations, if $w$ has degree at least 3, then we pretend that $v$ is saturated, and delete it before proceeding. We call such a leaf $v$ a discardable leaf. (We require $d(w) \geq 3$ so that we may delete $v$ without violating the induction hypothesis in the proof of Theorem 2.4, which requires that all undominated vertices have degree at least 2.) This imagined saturation of $v$ increases the value of the original move, which sometimes proves useful. Intuitively, since $v$ is of no use to either player and will eventually be saturated "for free", the move will eventually yield two points of value; we claim those extra points immediately. (Note that discarding $v$ can neither create nor destroy any linking vertices.) Refer to Figure 1, which illustrates a move of value 16: after the move at $x$, vertex $v$ is discardable, but $u$ is not, because not all of its neighbor's neighbors are dominated. (Note that there has been no net change in the number of linking vertices - one was created, while one was eliminated.)
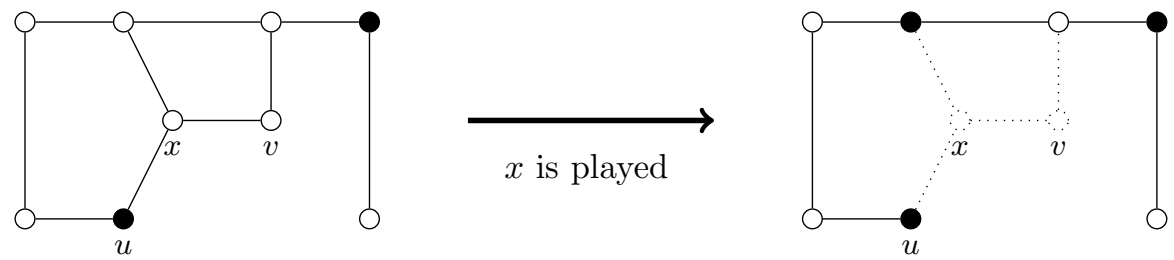

FiguRE 1. Empty vertices are undominated; filled vertices are dominated. Dotted vertices and edges have been removed from the graph.

Let $H_{1}, H_{2}$, and $H_{3}$ be the graphs $K_{1,2}, K_{1,3}$, and $K_{1,4}$, respectively, each with the center undominated and the leaves dominated, and let $H_{4}$ be $C_{4}$ with all vertices undominated; refer to Figure 2. We are now ready to prove Theorem 2.4. Recall its statement:

Theorem 2.4. Let $G$ be a reduced graph in which all undominated vertices have degree at least 2. If $G$ has $n$ vertices, of which $d$ are dominated, $s$ are saturated, and $\ell$ are linking 


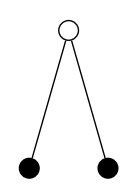

$H_{1}$

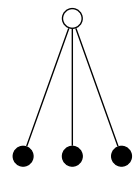

$\mathrm{H}_{2}$

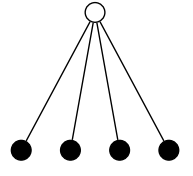

$\mathrm{H}_{3}$

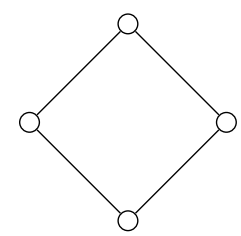

$H_{4}$

FiguRE 2. Empty vertices are undominated; filled vertices are dominated.

vertices, then

$$
\gamma_{g}(G) \leq \frac{6 n-4 d-2 s+\ell}{10} \quad \text { and } \quad \gamma_{g}^{\prime}(G) \leq \frac{6 n-4 d-2 s+\ell+5}{10} .
$$

Proof. We use induction on $n-s$. For the base case, suppose $G$ has $k$ components, each isomorphic to $H_{1}, H_{2}, H_{3}$, or $H_{4}$. No matter how Dominator and Staller play, exactly one move is used in each copy of $H_{1}, H_{2}$, and $H_{3}$, and two moves are used in each copy of $H_{4}$. For $i \in\{1,2,3,4\}$, let $k_{i}$ denote the number of components of $G$ isomorphic to $H_{i}$; now $n=s+3 k_{1}+4 k_{2}+5 k_{3}+4 k_{4}$, while $d=s+2 k_{1}+3 k_{2}+4 k_{3}$ and $\ell=0$. Now

$$
\begin{aligned}
\gamma_{g}(G) & \leq k_{1}+k_{2}+k_{3}+2 k_{4} \\
& =\frac{10 k_{1}+10 k_{2}+10 k_{3}+20 k_{4}}{10} \\
& =\frac{6 n-4 d-2 s-2 k_{2}-4 k_{3}-4 k_{4}}{10} \\
& \leq \frac{6 n-4 d-2 s+\ell}{10} ;
\end{aligned}
$$

Similarly, $\gamma_{g}^{\prime}(G) \leq \frac{6 n-4 d-2 s+\ell+5}{10}$.

Suppose now that $G$ has some component not isomorphic to $H_{1}, H_{2}, H_{3}$, or $H_{4}$. Recall that to each move of the game we assign a numeric value: a move that dominates $d^{\prime}$ vertices, saturates $s^{\prime}$ vertices, and changes the number of linking vertices by $\ell^{\prime}$ has value $4 d^{\prime}+2 s^{\prime}-\ell^{\prime}$. (Recall also that discardable leaves are viewed as being saturated; refer to Lemma 2.6.) The value of a move represents the change induced by that move in the numerator of the claimed bounds on $\gamma_{g}$ and $\gamma_{g}^{\prime}$. That is, suppose some player makes a move that dominates $d^{\prime}$ vertices, saturates $s^{\prime}$ vertices, and changes the number of linking vertices by $\ell^{\prime}$; let $\alpha=4 d^{\prime}+2 s^{\prime}-\ell^{\prime}$, and let $G^{\prime}$ be the resulting residual graph. Since all undominated vertices in $G^{\prime}$ still retain all neighbors they had in $G$, the induction hypothesis applies to $G^{\prime}$. Thus

$$
\gamma_{g}\left(G^{\prime}\right) \leq \frac{6 n-\left(4 d+4 d^{\prime}\right)-\left(2 s+2 s^{\prime}\right)+\left(\ell+\ell^{\prime}\right)}{10}=\frac{6 n-4 d-2 s+\ell}{10}-\frac{\alpha}{10}
$$

and

$(\star \star) \quad \gamma_{g}^{\prime}\left(G^{\prime}\right) \leq \frac{6 n-\left(4 d+4 d^{\prime}\right)-\left(2 s+2 s^{\prime}\right)+\left(\ell+\ell^{\prime}\right)+5}{10}=\frac{6 n-4 d-2 s+\ell+5}{10}-\frac{\alpha}{10}$.

Intuitively, the computations above suggest that Dominator wants the average value of every move played in the game to be at least 10, since then (on average) each move decreases the inductive bound by 1 , while adding 1 to the number of moves actually made. 
We now return to the game. Suppose Staller plays first. Let $\alpha$ denote the value of Staller's move; by Lemma 2.5, $\alpha \geq 5$. Letting $G^{\prime}$ denote the resulting residual graph, we have

$$
\gamma_{g}^{\prime}(G)=1+\gamma_{g}\left(G^{\prime}\right) \leq 1+\frac{6 n-4 d-2 s+\ell}{10}-\frac{\alpha}{10} \leq \frac{6 n-4 d-2 s+\ell+5}{10},
$$

where the first inequality follows from inequality $(\star)$.

Now suppose Dominator plays first. We consider several cases.

Case 1: $G$ has an undominated vertex with three or more undominated neighbors. Let $v$ be such a vertex, and suppose it has $k$ undominated neighbors, where $k \geq 3$. Dominator plays $v$. This dominates $k+1$ vertices and saturates at least one (namely, $v$ itself). The move may also increase the number of linking vertices by as much as $k$, since each undominated neighbor of $v$ could potentially become a linking vertex. Hence the move has value at least $4(k+1)+2-k$, which in turn is at least 15 . Letting $G^{\prime}$ denote the resulting residual graph, we have

$$
\gamma_{g}(G) \leq 1+\gamma_{g}^{\prime}\left(G^{\prime}\right) \leq 1+\frac{6 n-4 d-2 s+\ell+5}{10}-\frac{15}{10}=\frac{6 n-4 d-2 s+\ell}{10},
$$

where the second inequality follows from inequality $(\star \star)$.

We henceforth assume that every undominated vertex in $G$ has at most two undominated neighbors. Consequently, playing an undominated vertex $v$ cannot change any neighbor $w$ into a linking vertex, since this would require $w$ to have at least two undominated neighbors other than $w$. Thus, when our strategy calls for Dominator to play an undominated vertex, we need not worry about creating any linking vertices.

Case 2: $G$ has an undominated vertex with two undominated neighbors and one leaf neighbor. Let $v$ be such a vertex in $G$. Dominator plays $v$; this dominates $v$ and its undominated neighbors, and saturates $v$ and its leaf neighbor. Thus the move dominates at least three vertices and saturates at least two (and, as noted above, cannot increase the number of linking vertices), so its value is at least 16 . As before, letting $G^{\prime}$ denote the resulting residual graph, we have

$$
\gamma_{g}(G) \leq 1+\gamma_{g}^{\prime}\left(G^{\prime}\right) \leq 1+\frac{6 n-4 d-2 s+\ell+5}{10}-\frac{16}{10}<\frac{6 n-4 d-2 s+\ell}{10}
$$

as desired.

Case 3: Some component of $G$ not isomorphic to $H_{4}$ has an undominated vertex with two undominated neighbors. Let $v$ be such a vertex, and let $u$ and $w$ be its undominated neighbors; if possible, we choose $v$ in such a way that $u$ has no other undominated neighbors.

If $u$ has no undominated neighbors other than $v$, then Dominator plays $v$. This dominates $u, v$, and $w$, and saturates $u$ and $v$. Thus the move has value at least 16, and the claimed bound holds as above.

Otherwise, we may assume that $u$ has exactly two undominated neighbors, $v$ and (say) $t$. Similarly, $t$ must have two undominated neighbors, since otherwise we would have taken $v$ to be $u$. Continuing this reasoning, we find an induced cycle $C: v_{1} v_{2} \ldots v_{k}$ in $G$, where $v=v_{1}, u=v_{2}, w=v_{k}$, and each $v_{i}$ has exactly two undominated neighbors.

First, suppose that $k=3$ (that is, $u$ and $w$ are adjacent). In this case, Dominator plays $v$. This dominates and saturates $u, v$, and $w$, and hence has value at least 18 , so again the claimed bound holds. Hence, we may assume that $k \geq 4$. 
Next, suppose that $k=4$. Since the component containing $v$ is not isomorphic to $H_{4}$, one of the vertices $v_{i}$ on the cycle $C$ must have some dominated neighbor not on $C$. Without loss of generality, suppose $v_{1}$ has a dominated neighbor $x$. Dominator plays $v_{3}$. This move dominates $v_{2}, v_{3}$, and $v_{4}$ and saturates $v_{3}$; moreover, since the degree of $v_{1}$ is at least three and its neighbors are all dominated, $v_{2}$ becomes a discardable leaf, so we may imagine that $v_{2}$ has also been saturated (refer to Lemma 2.6). Thus, the move dominates three vertices and saturates at least two, so its value is at least 16, and the bound follows as usual. Hence, we may assume that $k \geq 5$.

Suppose that playing some vertex of $C$ creates a saturated vertex that does not belong to $V(C)$. Renaming vertices of $C$, if necessary, we may assume that $v$ is such a vertex of $C$ and that $x \notin V(C)$ becomes saturated when $v$ is played. Playing the move $v$ dominates $u$, $v$, and $w$, and saturates $v$ and $x$. Thus, the value of playing the move $v$ is at least 16 , and the bound follows. Hence, we may assume that playing a vertex of $C$ saturates no vertex except the vertex played.

By Cases 1 and 2, the graph $G$ contains no (dominated) leaf whose neighbor has two or more undominated neighbors. Suppose playing some vertex of $C$ creates a (dominated) leaf that does not belong to $V(C)$. Renaming vertices of $C$, if necessary, we may assume that $v$ is such a vertex of $C$ that creates a leaf, say $x$, not in $V(C)$. (Possibly, $x$ is a neighbor of $v$.) Immediately before the vertex $v$ is played, we note that the vertex $x$ is a dominated vertex (of degree at least 2) not on $C$ that is adjacent to at least one of $u, v$ and $w$. By our earlier assumption, the vertex $x$ is not saturated when $v$ is played. Playing the move $v$ dominates $u, v$, and $w$, and saturates $v$. Further, this move additionally reduces the number of linking vertices since the vertex $x$ is no longer a linking vertex. Thus, the value of playing the move $v$ is at least 15, and the bound follows. Hence, we may assume that playing a vertex of $C$ does not create a (dominated) leaf except for that vertex's two neighbors on $C$.

Dominator now plays $v$. This move dominates $u, v$, and $w$, and saturates $v$. The value of the move is therefore at least 14 . Let $G^{\prime}$ denote the resulting residual graph. By our earlier assumptions, playing the vertex $v$ creates no new leaves except for $u$ and $w$, neither of which has a neighbor with two or more undominated neighbors. Thus, by Lemma 2.5, $G^{\prime}$ has no moves of value 5, so Staller's ensuing move has value at least 6 . Thus, Dominator's move and Staller's response to his move have a combined total value of at least 20 . Letting $G^{\prime \prime}$ denote the residual graph after both moves, we have

$$
\gamma_{g}(G) \leq 2+\gamma_{g}\left(G^{\prime \prime}\right) \leq 2+\frac{6 n-4 d-2 s+\ell}{10}-\frac{20}{10}=\frac{6 n-4 d-2 s+\ell}{10},
$$

as needed.

By Cases 1, 2 and 3, we may now assume that no undominated vertex in a component of $G$ that is not isomorphic to $H_{4}$ has more than one undominated neighbor. We remark that for the remainder of the game, no new linking vertices can be produced: a linking vertex can arise only when an undominated vertex with two or more undominated neighbors becomes dominated, and clearly this can no longer happen.

Case 4: Some component of $G$ not isomorphic to $H_{1}, H_{2}$, or $H_{3}$ has a leaf. Let $v$ be some such leaf of $G$, and let $u$ be its neighbor. (Note that by assumption, $v$ must be dominated; since $G$ is a reduced graph, $u$ must be undominated.) By assumption, the vertex $u$ can have at most one undominated neighbor. 
If $u$ has an undominated neighbor, $t$, then Dominator plays $t$. This move dominates at least two vertices, namely $t$ and $u$, and saturates at least three vertices, namely $t, u$, and $v$. If this move ends the game, then $6 n-4 d-2 s=4(n-d)+2(n-s) \leq 4 \cdot 2+2 \cdot 3=14$, and so

$$
\gamma_{g}(G)=1<\frac{6 n-4 d-2 s}{10} \leq \frac{6 n-4 d-2 s+\ell}{10} .
$$

Suppose instead that the game continues after Dominator's move, and that Staller plays an optimal move in response. Staller's response dominates at least one vertex, saturates at least one, and, as noted above, cannot produce any new linking vertices; hence, this move has value at least 6. Thus, Dominator's and Staller's moves together have value at least 20. Letting $G^{\prime \prime}$ denote the resulting residual graph after these two moves, we have

$$
\gamma_{g}(G) \leq 2+\gamma_{g}\left(G^{\prime \prime}\right) \leq 2+\frac{6 n-4 d-2 s+\ell}{10}-\frac{20}{10}=\frac{6 n-4 d-2 s+\ell}{10} .
$$

Now suppose instead that $u$ has no undominated neighbors. By assumption, the degree of $u$ is at least 2 , so $u$ has at least one dominated neighbor aside from $v$. If $u$ is adjacent to five or more leaves, then Dominator plays $u$. This dominates one vertex and saturates at least six, and hence has value at least 16 , so the claimed bound on $\gamma_{g}(G)$ follows in the usual way. Otherwise, since the component containing $u$ is not isomorphic to $H_{1}, H_{2}$, or $H_{3}$, the vertex $u$ must have some non-leaf neighbor $t$. Dominator plays $t$, which dominates all undominated neighbors of $t$ (of which there must be at least two), and saturates at least three vertices (namely, $t, u$, and $v$ ). Moreover, this move eliminates the linking vertex $t$. Thus the value of the move is at least 15, and the bound once again follows.

Case 5: $G$ contains a dominated vertex with at least three undominated neighbors. Let $v$ be such a vertex. Dominator plays $v$. This dominates each neighbor of $v$, of which there are at least three; it also saturates $v$, and reduces the number of linking vertices by at least 1 (since $v$ itself was a linking vertex). Thus the value of the move is at least 15 , and the bound follows as usual.

We henceforth assume that every component of $G$ not isomorphic to $H_{1}, H_{2}$, or $H_{3}$ has minimum degree at least 2, for otherwise Case 4 applies and the desired result follows. Further, we assume that every dominated vertex in $G$ has degree exactly 2 (with both its neighbors undominated), for otherwise Case 5 applies and the desired result again follows. At this point, the structure of $G$ is determined. By assumption, $G$ has some component not isomorphic to $\mathrm{H}_{1}, \mathrm{H}_{2}, \mathrm{H}_{3}$, or $H_{4}$. In these components, each undominated vertex has at most one undominated neighbor and, consequently, at least one dominated neighbor.

Case 6: Some component of $G$ not isomorphic to $\mathrm{H}_{1}, \mathrm{H}_{2}, \mathrm{H}_{3}$, or $\mathrm{H}_{4}$ has an undominated vertex with an undominated neighbor. Let $v$ be such a vertex, let $u$ be its undominated neighbor, let $w$ be a dominated neighbor of $v$, and let $t$ be a dominated neighbor of $u$. Dominator plays $v$.

If $t=w$, then Dominator's move dominates $u$ and $v$, saturates $u, v$, and $t$, and eliminates the linking vertex $t$. Thus the move has value at least 15, and the bound follows as usual.

Otherwise, Dominator's move dominates and saturates both $u$ and $v$, and causes $t$ and $w$ to no longer be linking vertices. Thus, the move has value at least 14. As argued in Case 4, if this move ends the game, then the desired bound follows; otherwise, Staller's response 
must have value at least 6, so Dominator's move and Staller's response to his move have a combined total value of at least 20 , and the bound again follows.

We may assume in what follows that every neighbor of an undominated vertex in a component of $G$ that is not $H_{1}, H_{2}, H_{3}$, or $H_{4}$ is a dominated vertex, for otherwise Case 6 applies and the desired result follows. By assumption, $G$ has some component, $C$ say, not isomorphic to $H_{1}, H_{2}, H_{3}$, or $H_{4}$. Let $v$ be an undominated vertex in $C$. By assumption, all neighbors of $v$ are dominated and $v$ has at least two neighbors. Let $u$ and $w$ be (dominated) neighbors of $v$, and let $t$ be the other undominated neighbor of $u$. Dominator plays $u$, which dominates $t$ and $v$, saturates $u, v$ and $t$, and causes $u$ and $w$ to no longer be linking vertices. Thus, the move has value at least 16, and the claimed bound follows. This completes the proof of Theorem 2.4.

Theorem 2.4 immediately implies our next main result.

Theorem 2.7. Let $G$ be an $n$-vertex graph. If $G$ has minimum degree at least 2 , then

$$
\gamma_{g}(G) \leq \frac{3 n}{5} \quad \text { and } \quad \gamma_{g}^{\prime}(G) \leq \frac{3 n-1}{5}
$$

Proof. At the beginning of the game, $G$ has no dominated vertices, no saturated vertices, and no linking vertices. Thus, by Theorem 2.4 ,

$$
\gamma_{g}(G) \leq \frac{6 n-4 \cdot 0-2 \cdot 0+0}{10}=\frac{6 n}{10}=\frac{3 n}{5} .
$$

Now consider the Staller-start game. Suppose Staller's initial move dominates $d^{\prime}$ vertices, saturates $s^{\prime}$, and creates $\ell^{\prime}$ linking vertices. As usual, we have $s^{\prime} \geq 1$. Since $G$ has minimum degree at least 2 , we also have $d^{\prime} \geq 3$. Finally, since the vertex at which Staller played cannot become a linking vertex, we have $\ell^{\prime} \leq d^{\prime}-1$. Letting $G^{\prime}$ denote the residual graph after Staller's first move, we have

$$
\begin{aligned}
\gamma_{g}^{\prime}(G) & \leq 1+\gamma_{g}\left(G^{\prime}\right) \\
& \leq 1+\frac{1}{10}\left(6 n-4 d^{\prime}-2 s^{\prime}+\ell^{\prime}\right) \\
& \leq 1+\frac{1}{10}\left(6 n-3 d^{\prime}-2 s^{\prime}-1\right) \\
& \leq 1+\frac{1}{10}(6 n-3 \cdot 3-2 \cdot 1-1) \\
& =\frac{6 n-2}{10} \\
& =\frac{3 n-1}{5}
\end{aligned}
$$

as claimed.

Thus we have established the 3/5-Conjecture for all graphs with minimum degree at least 2 . We remark that Theorem 2.4 can be leveraged to establish stronger bounds on $\gamma_{g}(G)$ and $\gamma_{g}^{\prime}(G)$ when $\delta(G)>2$. However, in this context, Bujtás has recently obtained even better bounds using different arguments; we refer the interested reader to [9].

\section{EDGE-REMOVAL}

In this section, we establish another bound on $\gamma_{g}$ and $\gamma_{g}^{\prime}$ that sometimes strengthens Theorem 2.2. Before proving this bound, we first answer a question posed by Brešar, Dorbec, Klavžar, and Košmrlj in [2]. They proved the following: 
Theorem 3.1 ([2], Theorem 2.1). If $G$ is a graph and $e \in E(G)$, then

$$
\left|\gamma_{g}(G)-\gamma_{g}(G-e)\right| \leq 2 \quad \text { and } \quad\left|\gamma_{g}^{\prime}(G)-\gamma_{g}^{\prime}(G-e)\right| \leq 2 .
$$

This result motivated them to pose the following problem:

Problem 3.2 ([2], Problem 4.1). Given a positive integer $k$, can one find a general upper and lower bound for $\gamma_{g}(G)-\gamma_{g}\left(G_{k}\right)$, where $G_{k}$ is obtained from a graph $G$ by deletion of $k$ edges from $G$ ?

Our next main result is a solution to Problem 3.2. For this result, we need a notion that is more restrictive than that of a "partially-dominated graph". Given a graph $G$ and a set $X \subseteq V(G)$, we define $\gamma_{g}(G ; X)=\gamma_{g}(G \mid N[X])$ and $\gamma_{g}^{\prime}(G ; X)=\gamma_{g}^{\prime}(G \mid N[X])$. That is, $\gamma_{g}(G ; X)$ (resp. $\left.\gamma_{g}^{\prime}(G ; X)\right)$ represents the number of turns remaining in the game on $G$ in which the vertices of $X$ have been played thus far, and Dominator (resp. Staller) has the next turn. We will also need the following result, proved partially in [5] and partially in [20].

Theorem 3.3 ([5, 20]). If $G$ is a partially-dominated graph, then $\left|\gamma_{g}(G)-\gamma_{g}^{\prime}(G)\right| \leq 1$.

We remark that Theorem 3.3 was originally stated for ordinary graphs, but holds more generally for partially-dominated graphs. (It is an easy consequence of the Continuation Principle, which applies in this greater generality.)

We are now ready to prove our next result. Recall that we use the notation $S \triangle T$ to denote the symmetric difference of $S$ and $T$.

Theorem 3.4. If $G$ and $H$ are graphs on a common vertex set $V$, then

$$
\left|\gamma_{g}(G)-\gamma_{g}(H)\right| \leq k+\epsilon \quad \text { and } \quad\left|\gamma_{g}^{\prime}(G)-\gamma_{g}^{\prime}(H)\right| \leq k+\epsilon,
$$

where $k=|E(G) \triangle E(H)|$, and $\epsilon$ is 0 if $k$ is even and 1 if $k$ is odd.

Proof. By symmetry, it suffices to show

$$
\gamma_{g}(G)-\gamma_{g}(H) \leq k+\epsilon \quad \text { and } \quad \gamma_{g}^{\prime}(G)-\gamma_{g}^{\prime}(H) \leq k+\epsilon
$$

To prove (1), we give a Dominator strategy for the game on $G$. Dominator "imagines" a game on $H$, and uses an optimal strategy for that game to guide his play in the game on $G$. We refer to the game on $G$ as the primary game and to the game on $H$ as the secondary game. Throughout, we occasionally play moves in the primary game but not the secondary game; conversely, we ensure that every move played in the secondary game has been played in the primary game as well. In order to maintain the hypothesis that both games operate on a common vertex set, we abuse notation slightly: when we refer to the "residual graph", we forego discarding saturated vertices (though we do still discard edges with both endpoints dominated).

Before attempting to prove (1) in general, we show that we may reduce to the case where $E(G) \triangle E(H)$ is a matching. For the sake of reaching a contradiction, let $(G, H)$ be a counterexample to (1) having the minimum possible number of unsaturated vertices, and let $v$ be some vertex incident to two or more edges of $E(G) \triangle E(H)$. If Dominator has the first turn, then he plays on $v$ in both games; let $G^{\prime}$ and $H^{\prime}$ be the residual graphs, let $k^{\prime}=\left|E\left(G^{\prime}\right) \triangle E\left(H^{\prime}\right)\right|$, and let $\epsilon^{\prime}$ be 0 if $k^{\prime}$ is even and 1 if $k^{\prime}$ is odd. Since $k^{\prime} \leq k-2$, we 
have $k^{\prime}+\epsilon^{\prime} \leq k+\epsilon-2$. Now

$$
\begin{aligned}
\gamma_{g}(G) & \leq 1+\gamma_{g}^{\prime}\left(G^{\prime}\right) \\
& \leq 1+\gamma_{g}^{\prime}\left(H^{\prime}\right)+k^{\prime}+\epsilon^{\prime} \\
& \leq 1+\left(\gamma_{g}\left(H^{\prime}\right)+1\right)+k^{\prime}+\epsilon^{\prime} \\
& \leq \gamma_{g}\left(H^{\prime}\right)+k+\epsilon \\
& \leq \gamma_{g}(H)+k+\epsilon,
\end{aligned}
$$

where the first inequality holds because Dominator does at least as well by playing optimally as by playing $v$, the second holds by minimality of $(G, H)$, the third follows from Theorem 3.3, and the last follows from the Continuation Principle.

Suppose instead that Staller has the first turn, and plays on some vertex $x$ in the primary game; Dominator imagines that Staller played $x$ in the secondary game as well. Regardless of Staller's choice of $x$, Dominator plays $v$ in both games. As before, let $G^{\prime}$ and $H^{\prime}$ be the residual graphs, let $k^{\prime}=\left|E\left(G^{\prime}\right) \triangle E\left(H^{\prime}\right)\right|$, and let $\epsilon^{\prime}$ be 0 if $k^{\prime}$ is even and 1 if $k^{\prime}$ is odd. Since $k^{\prime} \leq k-2$, we have $k^{\prime}+\epsilon^{\prime} \leq k+\epsilon-2$. Now

$$
\gamma_{g}^{\prime}(G) \leq 2+\gamma_{g}^{\prime}\left(G^{\prime}\right) \leq 2+\gamma_{g}^{\prime}\left(H^{\prime}\right)+k^{\prime}+\epsilon^{\prime} \leq \gamma_{g}^{\prime}\left(H^{\prime}\right)+k+\epsilon \leq \gamma_{g}^{\prime}(H)+k+\epsilon .
$$

Hence we may assume that $E(G) \triangle E(H)$ is a matching. We refer to the elements of $E(G) \triangle E(H)$ as volatile edges. Throughout the game, we classify the volatile edges as follows:

- resolved edges are those with both endpoints dominated in the primary game.

- protected edges are those with one endpoint played in the primary game, the other undominated in the primary game, and neither endpoint played in the secondary game.

- dangerous edges are those with one endpoint played in both games and the other undominated in the primary game.

- untouched edges are those with neither endpoint played in either game and at least one endpoint undominated in the primary game.

Since we will maintain the property that every vertex played in the secondary game has in fact been played in both games, these classes partition the volatile edges. Note that once an edge becomes resolved, it permanently remains so. Similarly, once an edge ceases to be untouched, it can never return to being untouched. Finally, note that a dangerous edge cannot belong to $E(G)$, and hence must belong to $E(H)$; consequently, both endpoints must be dominated in the secondary game.

As noted, we aim to prove (1). In fact, we prove a stronger statement. Fix $X, Y \subseteq V$, and consider a scenario in which $X$ is the set of vertices played in the secondary game, while $X \cup Y$ is the set of vertices played in the primary game. Let $\ell$ denote the total number of untouched and dangerous edges, and let $\epsilon$ be 0 if $\ell$ is even and 1 if $\ell$ is odd. We claim that

$$
\gamma_{g}(G ; X \cup Y) \leq \gamma_{g}(H ; X)+\ell+\epsilon \quad \text { and } \quad \gamma_{g}^{\prime}(G ; X \cup Y) \leq \gamma_{g}^{\prime}(H ; X)+\ell+\epsilon ;
$$

note that taking $X=Y=\varnothing$ yields the original claim. If there are no undominated vertices in the primary game, then the primary game has ended, all edges are resolved, and the inequalities hold. Thus we may assume that the primary game is not yet complete. We proceed through induction on the number of undominated vertices in the secondary game. 
For the base case, suppose there are no undominated vertices in the secondary game. In the primary game, each remaining undominated vertex $x$ must lie on a dangerous edge: some neighbor of $x$ in $H$ must have been played in the secondary game (and hence in both games) without dominating $x$ in the primary game. Moreover, there are no untouched edges: since every volatile edge $u v$ is incident to no other volatile edges, the only way for one endpoint (say, $v$ ) to be dominated in the secondary game but not in the primary game is to have $u \in X$ and $u v \notin E(G)$. Consequently, at most $\ell$ undominated vertices remain in $G$, hence $\gamma_{g}(G ; X \cup Y) \leq \ell$ and $\gamma_{g}^{\prime}(G ; X \cup Y) \leq \ell$.

Now suppose that both games are still ongoing, and it is Dominator's turn. Dominator chooses an optimal move $x$ in the secondary game. If $x \notin Y$, or if $x \in Y$ but $x$ lies on no volatile edge, then Dominator plays $x$ in both games. (Recall that we have modified the rules of the game so that all Dominator moves are "legal", even repeated moves; so that we may apply the induction hypothesis, we may require without loss of generality that $x$ dominates at least one new vertex in the secondary game, but need not worry about whether it dominates anything new in the primary game.) This move cannot cause any protected edges to become dangerous, so the total number of untouched and dangerous edges does not increase. Hence

$$
\begin{aligned}
\gamma_{g}(G ; X \cup Y) & \leq 1+\gamma_{g}^{\prime}(G ; X \cup Y \cup\{x\}) \\
& \leq 1+\gamma_{g}^{\prime}(H ;(X \cup\{x\}))+\ell+\epsilon \\
& =\gamma_{g}(H ; X)+\ell+\epsilon .
\end{aligned}
$$

If instead $x \in Y$ and $x$ lies on volatile edge $x y$, then Dominator plays $x$ in the secondary game as before, but now plays $y$ in the primary game. This makes edge $x y$ resolved. Moreover, no other volatile edges can be incident to $y$; hence, as before, this move cannot increase the total number of untouched and dangerous edges. Let $Y^{\prime}=(Y \cup\{y\}) \backslash\{x\}$. As before,

$$
\begin{aligned}
\gamma_{g}(G ; X \cup Y) & \leq 1+\gamma_{g}^{\prime}\left(G ; X \cup Y^{\prime} \cup\{x\}\right) \\
& \leq 1+\gamma_{g}^{\prime}(H ; X \cup\{x\})+\ell+\epsilon \\
& =\gamma_{g}(H ; X)+\ell+\epsilon .
\end{aligned}
$$

Next suppose that it is Staller's turn, and that Staller plays $x$ in the primary game. If this is a legal move in the secondary game, then Dominator imagines that Staller played $x$ there as well. We claim that this move cannot make any protected edges dangerous. Let $u v$ be a protected edge, and let $v$ be the endpoint not dominated in the primary game. By definition of "protected", $u$ has not been played in the secondary game. Additionally, since $v$ lies on no volatile edge aside from $u v$, it must be that $v$ is undominated in the secondary game (as any move that dominated $v$ in the secondary game would have dominated it in the primary game as well). If Staller's move dominates $v$ in the secondary game, then it does so in the primary game as well, and $u v$ becomes resolved; otherwise, $u v$ cannot be dangerous, since both endpoints of every dangerous edge are dominated in the secondary game. Thus no protected edge becomes dangerous, so the total number of untouched and dangerous edges 
does not increase. Consequently,

$$
\begin{aligned}
\gamma_{g}^{\prime}(G ; X \cup Y) & =1+\gamma_{g}(G ; X \cup Y \cup\{x\}) \\
& \leq 1+\gamma_{g}(H ; X \cup\{x\})+\ell+\epsilon \\
& \leq \gamma_{g}^{\prime}(H ; X)+\ell+\epsilon .
\end{aligned}
$$

Suppose instead that Staller's move is not legal in the secondary game. In this case, Dominator may not imagine Staller making this move in the secondary game as well. Instead, he views this move as having been played only in the primary game, and must respond only in the primary game. Dominator must take care to choose a productive response, since he wants to minimize the number of moves played in the primary game but not in the secondary game.

This situation can only arise in a few ways. Let $y$ be some vertex dominated by Staller's move in the primary game. Clearly $x y \in E(G)$, and neither $x$ nor $y$ had already been played in either game. If $x y \notin E(H)$, then $x y$ is volatile; consequently, since $y$ was not already dominated in the primary game, and since $y$ can lie on only one volatile edge, $y$ must be undominated in the secondary game. Thus, Staller's move transforms $x y$ from an untouched edge to a protected edge, and hence decreases the number of untouched edges.

Suppose instead that $x y \in E(H)$. Since Staller's move was not legal in the secondary game, it must be that $y$ was already dominated in the secondary game. Thus, $y$ must lie on some volatile edge $y z$, where $y z \in E(H) \backslash E(G)$, and $z$ has already been played in the secondary game (and hence also in the primary game). Such an edge must be dangerous, and Staller's move makes it resolved. Thus the number of dangerous edges decreases.

If at least one other dangerous or untouched edge remains, then Dominator chooses some undominated endpoint $v$ of such an edge and plays it in the primary game. (Note that neither $x$ nor $v$ gets played in the secondary game; these moves are made only in the primary game.) The volatile edge in question becomes either protected or resolved. In addition, as argued above, Staller's move also reduces the total number of untouched and dangerous edges. Hence the total number of untouched and dangerous edges decreases by at least 2, so

$$
\begin{aligned}
\gamma_{g}^{\prime}(G ; X \cup Y) & \leq 2+\gamma_{g}^{\prime}(G ; X \cup Y \cup\{x, y\}) \\
& \leq 2+\gamma_{g}^{\prime}(H ; X)+(\ell+\epsilon-2) \\
& \leq \gamma_{g}^{\prime}(H ; X)+\ell+\epsilon .
\end{aligned}
$$

as desired. If instead no other dangerous or untouched edges remain, then Dominator plays any arbitrary move $y$ in the primary game. In this case $\ell+\epsilon=2$, so

$$
\gamma_{g}^{\prime}(G ; X \cup Y) \leq 2+\gamma_{g}^{\prime}(G ; X \cup Y \cup\{x, y\}) \leq 2+\gamma_{g}^{\prime}(H ; X)=\gamma_{g}^{\prime}(H ; X)+\ell+\epsilon,
$$

which completes the proof.

Theorem 3.4 is tight for all values of $k$, as exhibited by the following examples. Let $G_{k}=K_{3,3} \cup K_{1,2 k+2}$ and let $H_{k}$ be obtained from $G$ by deleting $k$ edges from the copy of $K_{1,2 k+2}$, and so $H_{k}=K_{3,3} \cup K_{1, k+2} \cup k K_{1}$. Now $G_{k}$ and $H_{k}$ differ in precisely $k$ edges, and it is straightforward to verify that $\gamma_{g}\left(G_{k}\right)=3$, while $\gamma_{g}\left(H_{k}\right)=k+4$ when $k$ is odd, and $\gamma_{g}\left(H_{k}\right)=k+3$ when $k$ is even. Similarly, we have $\gamma_{g}^{\prime}\left(G_{k}\right)=4, \gamma_{g}\left(H_{k}\right)=k+5$ when $k$ is odd, and $\gamma_{g}\left(H_{k}\right)=k+4$ when $k$ is even. In lieu of a formal proof, we offer the following informal explanation. Neither player wants to play first on the component isomorphic to $K_{3,3}$. In the Dominator-start game, Dominator plays his first move on the center of the 
star, and afterwards, players alternately dominate isolated vertices. In $H_{k}$, when $k$ is odd, Staller is forced to play first on the $K_{3,3}$ component, and only two moves are played there; when $k$ is even, Dominator is forced to play first in that component, and three moves are played there. In the Staller-start game the strategies are similar, except that Staller begins by playing some leaf of the star. We remark that the same ideas can be used to construct connected graphs $G_{k}^{\prime}$ and $H_{k}^{\prime}$ witnessing tightness in Theorem 3.4; we omit the details.

Returning our attention to the 3/5-Conjecture, we note that Theorems 2.7 and 3.4 together yield a bound on $\gamma_{g}$ and $\gamma_{g}^{\prime}$ that, in some cases, is stronger than Theorem 2.2.

Corollary 3.5. If $G$ is an $n$-vertex isolate-free graph with $\ell$ vertices of degree 1 , then $\gamma_{g}(G) \leq$ $\frac{3 n}{5}+\left\lceil\frac{\ell}{2}\right\rceil+1$ and $\gamma_{g}^{\prime}(G) \leq \frac{3 n-1}{5}+\left\lceil\frac{\ell}{2}\right\rceil+1$.

Proof. If $G=K_{2}$, then the claim is clear. Otherwise, we may augment $G$ to a graph $H$ of minimum degree at least 2 by adding at most $\lceil\ell / 2\rceil$ edges. By Theorem 2.7,

$$
\gamma_{g}(H) \leq \frac{3 n}{5} \quad \text { and } \quad \gamma_{g}^{\prime}(H) \leq \frac{3 n-1}{5}
$$

Hence by Theorem 3.4,

$$
\gamma_{g}(G) \leq \gamma_{g}(H)+\left\lceil\frac{\ell}{2}\right\rceil+1 \leq \frac{3 n}{5}+\left\lceil\frac{\ell}{2}\right\rceil+1
$$

and

$$
\gamma_{g}^{\prime}(G) \leq \gamma_{g}(H)+\left\lceil\frac{\ell}{2}\right\rceil+1 \leq \frac{3 n-1}{5}+\left\lceil\frac{\ell}{2}\right\rceil+1
$$

To close the paper, we comment on Problem 4.2 in [2], which asks which subsets of $\{-2,-1,0,1,2\}$ can be realized as $\left\{\gamma_{g}(G)-\gamma_{g}(G-e): e \in E(G)\right\}$ for some graph $G$. Theorem 3.4 yields a partial answer: each such set must be contained in $\{-2,-1,0\},\{-1,0,1\}$, or $\{0,1,2\}$, because for all edges $e, e^{\prime} \in E(G)$, we have $\left|\gamma(G-e)-\gamma\left(G-e^{\prime}\right)\right| \leq 2$.

\section{ACKNOWLEDGMENTS}

The authors would like to thank Boštjan Brešar, Sandi Klavžar, Gašper Košmrlj, and Douglas Rall for helpful discussions during a recent workshop on game domination hosted by Douglas Rall at Furman University. The authors would also like to thank the anonymous referees, whose insightful comments greatly improved the paper.

\section{REFERENCES}

[1] A. Bonato, W.B. Kinnersley, and P. Prałat, Game toppling number for complete and random graphs, Discrete Math. Theor. Comp. Sci. 16 (2014), 229-252.

[2] B. Brešar, P. Dorbec, S. Klavžar, and G. Košmrlj, Domination game: effect of edge- and vertex-removal, Discrete Math. 330 (2014), 1-10.

[3] J. Butterfield, T. Grauman, W.B. Kinnersley, K.G. Milans, C. Stocker, and D.B. West, On-line Ramsey theory for bounded-degree graphs, Electron. J. Combin. 18 (2011), Paper P136.

[4] B. Brešar, S. Klavžar, G. Košmrlj, and D.F. Rall, Domination game: extremal families of graphs for the 3/5-conjectures, Discrete Appl. Math. 161 (2013), 1308-1316.

[5] B. Brešar, S. Klavžar, and D. Rall, Domination game and an imagination strategy, SIAM J. Discrete Math. 24 (2010), 979-991. 
[6] B. Brešar, S. Klavžar, and D. Rall, Domination game played on trees and spanning subgraphs, Discrete Math. 313 (2013), 915-923.

[7] Cs. Bujtás, Domination game on trees without leaves at distance four, Proceedings of the 8th JapaneseHungarian Symposium on Discrete Mathematics and Its Applications (A. Frank, A. Recski, G. Wiener, eds.), June 4-7, 2013, Veszprém, Hungary, 73-78.

[8] C. Bujtás, personal communication.

[9] C. Bujtás, On the game domination number of graphs with given minimum degree, preprint, http://arxiv.org/abs/1406.7372.

[10] D.W. Cranston, W.B. Kinnersley, S. O, and D.B. West, Game matching number of graphs, Discrete Applied Math. 161 (2013), 1828-1836.

[11] T. Dinski and X. Zhu, Game chromatic number of graphs, Discrete Math. 196 (1999), 109-115.

[12] M. Gardner, Mathematical games, Scientific American 244 (1981), 18-26.

[13] J.A. Grytczuk, M. Hałuszczak, and H.A. Kierstead, On-line Ramsey theory, Electron. J. Combin. 11 (2004), Paper \#R57, 10 pp.

[14] J. A. Grytczuk, H. A. Kierstead, and P. Prałat, On-line Ramsey numbers for paths and stars, Discrete Math. Theor. Comp. Sci. 10 (2008), 63-74.

[15] T. W. Haynes, S. T. Hedetniemi, and P. J. Slater, Fundamentals of domination in graphs. Monographs and Textbooks in Pure and Applied Mathematics 208 (Marcel Dekker, 1998).

[16] H. A. Kierstead and A. V. Kostochka, Efficient graph packing via game colouring, Combin. Probab. Comput. 18 (2009), 765-774.

[17] G. Košmrlj, Realizations of the game domination number, J. Combin. Opt. 28 (2014), 447-461.

[18] H. A. Kierstead and T. Trotter, Planar graph coloring with an uncooperative partner, J. Graph Theory 18 (1994), 569-584.

[19] W. B. Kinnersley and P. Prałat, Game brush number, submitted.

[20] W. B. Kinnersley, D. B. West, and R. Zamani, Extremal problems for game domination number, SIAM J. Discrete Math. 27 (2013), 2090-2107

[21] U. Schauz, Mr. Paint and Mrs. Correct, Electron. J. Combin. 16 (2009), no. 1, Research Paper 77, 18 pp.

[22] X. Zhu, Game coloring the Cartesian product of graphs, J. Graph Theory 59 (2008), 261-278.

[23] X. Zhu, On-line list colouring of graphs, Electron. J. Combin. 16 (2009), no. 1, Research Paper 127, 16 pp.

Department of Pure and Applied Mathematics, University of Johannesburg, Auckland Park, 2006, South Africa

E-mail address: mahenning@uj.ac.za

Department of Mathematics, University of Rhode Island, Kingston, Ri 02881, United States of AmERica

E-mail address: billk@uri.edu 